\title{
Cleaning Performance of Megasonic Multi- Frequency Operating in Thickness Mode Transducers
}

\author{
Vetrimurugan, M.J. Goodson, Terry Lim and T. Sebastian
}

\begin{abstract}
A multi-frequency megasonic system (360/470 kHz) with thickness mode transducers was developed to remove the nano-dimensional and sub-micron particles more effectively from various surfaces and the results obtained are compared with the conventional megasonic system operating with only single frequency. The multi-frequency transducers are piezoelectric transducers operating in thickness mode at different operating frequencies. To assess cleaning ability, the maximum cleaning potential tests were performed and almost $30 \%$ cleaning improvement was achieved as compared to 470 $\mathrm{kHz}$ and almost $4 \%$ improvement was achieved as compared to $360 \mathrm{kHz}$ megasonic system operating with thickness mode transducers for the parts tested. Uniformity of the multifrequency megasonic cleaning system was measured using ppbTM probe. The results revealed that the cavitation activity was more uniform throughout the entire cleaning system even at the edges and corners of the system employing multifrequency. The uniformity of the multi-frequency megasonic system was also studied by using Liquid Particle Counting (LPC) technique. The results obtained are quite consistent even at various locations of the tank employing multi-frequency with thickness mode transducers. This study also covered run to run variations of the megasonic system. Multiple extraction study was also performed for various megasonic frequencies to measure the surface cleanliness (SC), maximum cleaning potential (MCP) and also to see the erosion propensity.
\end{abstract}

Keywords - multiple megasonic frequency, megasonic sweeping, thickness mode transducers, cavitation intensity, particle removal, nano, sub-micron

\section{Introduction}

Megasonic processing involves generating and using high frequency energy at frequencies above $280 \mathrm{kHz}$. There are two basic particle-removal mechanisms in acoustic fields: cavitation, which predominates at lower frequencies $(<132 \mathrm{kHz})$, and acoustic streaming, which is predominant at higher frequencies $(>280 \mathrm{kHz})[1]$. Acoustic streaming is

R. Vetrimurugan is with Crest Ultrasonics, Penang, Malaysia (phone: +012-583 7018; fax: +604-507 0018;

Terry Lim is with Crest Ultrasonics, Penang, Malaysia.

M.J. Goodson is with Crest Ultrasonics, Trenton, USA.

Sebastian is with Crest Ultrasonics, Trenton, USA. a time-independent fluid motion caused by the attenuation of sound wave travelling in the viscous fluid [2]. Acoustic streaming reduces the thickness of hydrodynamic boundary layer on the surface, due to which smaller size particles are exposed to large velocity gradients that leads to drag forces and rolling moments which subsequently overcome the adhesion force between particle and surface [3]. In megasonic process the piezoelectric transducers are excited by an alternating current signal that causes alternating expansion and contraction of the transducers; primarily the expansion and contraction changes the thickness of the transducers. The changes in thickness of the piezoelectric transducers generate the acoustic filed in the cleaning tank.

Megasonic cleaning system is typically used to clean delicate parts such as semiconductor wafers, disk drive components and high end glass substrates. A continuing trend in the disk drive industry is that cleanliness standards are becoming more stringent and precisely defined. These tightened cleaning standards are required for each and every components of the disk drive [4-5] to avoid any catastrophic failure of the disk drives. In general higher cleanliness with low sigma is the desired criteria to achieve more reliable products. To achieve higher cleanliness level and low sigma megasonic system with multi-frequency would be one of the options.

Megasonic system operating in multi-frequency with thickness mode transducers can helps to remove wide ranges of particle sizes including nano-dimensional and sub-micron particles as compared to the conventional system operating with single megasonic frequency. Kim et al., [6] have studied the effect of multi-frequency megasonic spot shower systems on removal of nano-dimensional particles from semiconductor wafer surfaces. The Particle Removal Efficiency (PRE) was improved almost 56.1\%.

In this study, a multi-frequency megasonic system $(360 / 470 \mathrm{kHz})$ with thickness mode transducers was developed to remove the nano-dimensional and sub-micron particles more effectively from various surfaces and the results obtained are compared with the conventional megasonic system operating with only single frequency. 


\section{Experimental Details}

All experiments were performed in Class 1000 Cleanroom of the Advanced Ceramics Technology Lab (ACT Lab), Malaysia. For this study, Crest Megasonic standard tanks with dimensions 10"x14" with bottom mounted transducers were used. The purpose of this experimental study was mainly to investigate the cleaning performance of multi-frequency operating in thickness mode transducers. This study relates megasonic tanks and associated methods involving two or more piezoelectric transducers operating in thickness mode at different megasonic frequencies such as $360 \mathrm{kHz}$ and $470 \mathrm{kHz}$ and also relates more particularly to improving the cleaning performance by matching the fundamental resonant or higher-level harmonic frequencies of multi-frequency transducers to the fundamental resonant or higher-level harmonic frequencies of a plate upon which the transducers are mounted.

To demonstrate the cleaning performance multiple extraction experiments were performed for various frequencies such as $360 \mathrm{kHz}, 470 \mathrm{kHz}$ and multi-frequency $360 / 470 \mathrm{kHz}$. The effect of various frequencies on removal of nano-dimensional particles, run to run variations of cleaning process and uniformity of the cleaning tank was also studied. The watt density used for $360 \mathrm{kHz}, 470 \mathrm{kHz}$ and $360 / 470 \mathrm{kHz}$ is 57 watts/lit, 46 watts/litter and 57 watts/lit respectively.

The $\mathrm{ppb}^{\mathrm{TM}}$ meter and Liquid Particle Counting (LPC) technique was used to measure the uniformity of the cleaning tank. The $\mathrm{ppb}^{\mathrm{TM}}$ probe is an instrument used to measure the energy density in units $\mathrm{W} / \mathrm{in}^{2}$ of cavitation in liquids [7]. The second physical mechanism, acoustic streaming was not measured in this study, but was visualized. The acoustic streaming pattern and thickness mode piezoelectric transducers arrangements are as shown in Figs. 1 and 2 respectively. In liquid particle counting technique the parts are placed at various locations of the multi-frequency tank and the resultant particles removed are measured. The variables such as temperature of the DeIonized (DI) water and dissolved oxygen (DO) level were maintained constant for each experiment. The components used for this study was aluminum metal spacer and e-coated disk separator.

In this study LiQuilaz SO2 particle measuring system $\left(\mathrm{PMS}^{\mathrm{TM}}\right.$ ) was used to measure the particle counts in the DI water and $>0.3 \mu \mathrm{m}$ particle size was reported. This study is also applicable for removal of nano-dimensional particles and removal of particles from other components as well. The experiments were repeated several times and the average of this value was taken to calculate the cleaning efficiency. Maximum cleaning potential with lower asymptote value is the desired function to select the best cleaning process [8].

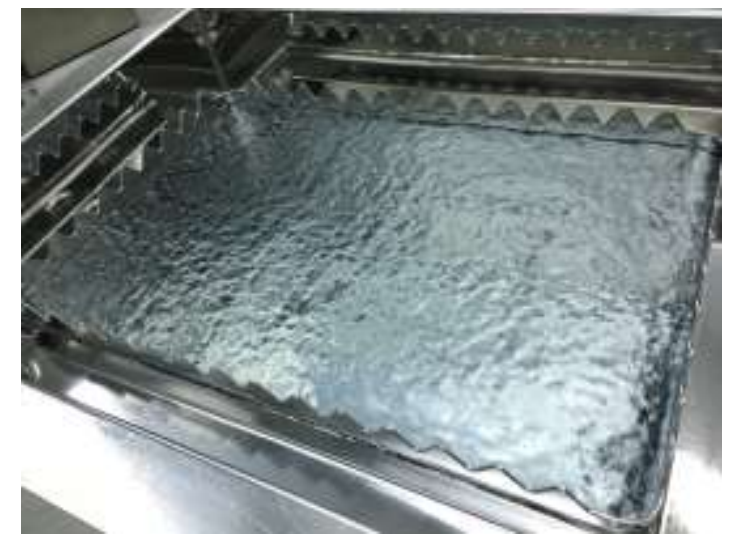

Fig. 1 Visualization of cavitation activity in 360/470 kHz multifrequency megasonic system operating in thickness mode transducers

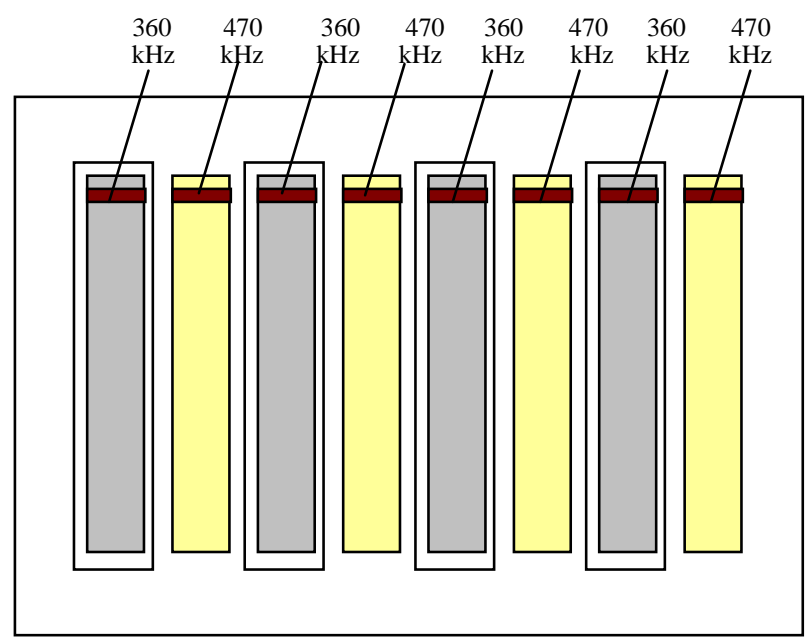

Fig. 2 Configuration of thickness mode piezoelectric multifrequency transducers bonded at the bottom of the tank

The configuration of thickness mode piezoelectric multifrequency transducers bonded at the bottom of the tank is shown in Fig.2. The thickness of the mounting plate at the transducer location is selected to provide a resonant frequency matched to that of its corresponding transducer. In this case two megasonic transducers are bonded to the same radiating surface [9]. As shown in Fig. 2, the $360 \mathrm{kHz}$ and $470 \mathrm{kHz}$ transducers are rectangular in shape and are arranged parallel to each other in an alternating sequence. Preferably, the transducers cover at least $80 \%$ portion of the bottom radiating surface of the tank. It is desirable to generate more uniform energy throughout the tank. Covering a high percentage of the surface area of the tank bottom with transducers ensures that the megasonic energy transferred to the fluid is relatively uniform.

In multi frequency megasonic process the resonant frequency of the mounting plate is matched to the operating frequency of the transducers. Such a multi-frequency array provides a sequence of megasonic frequencies in the tank. The advantage of this multi-frequency is that it substantially reduces the risk associated with handling of parts being processed and also helps to improve the overall cleaning performance. Matching the mounting plate frequencies to 
the multi-frequency transducers is an efficient way to transfer energy, which create improved and stronger microstreaming activity which improves the efficiency of microstreaming cleaning and other uses of micro-streaming.

\section{Results and Discussion}

\section{A. Megasonic Power Distribution for Multi-Frequency Operating in Thickness Mode Transducers}

The power distribution in a cleaning vessel measured by $\mathrm{ppb}^{\mathrm{TM}}$ meter for multi-frequency operating in thickness mode transducer is shown in Fig. 3. The result shows that the power is more uniformly distributed throughout the entire cleaning vessel. When the multi-frequency 360/470 $\mathrm{kHz}$ is swept at predetermined rate each transducer will vibrate at their optimum resonant frequency [10] and thus eliminates traditional power surges and establishes more uniform activity in a cleaning vessel even at the corners and edges of the system.

The uniformity of the cleaning tank was also checked by using LPC technique. The results obtained for aluminum spacer component cleaned at various locations of the tank is shown in Fig. 4. The result shows that the particle removal is more uniform throughout the entire cleaning vessel even for the megasonic system operating with multi-frequency. Even the corners and edges of tanks in systems employing "megasonic sweeping" display particle removal almost equivalent to those prevailing at the center, in the plane perpendicular to the transducer. The presence of sweep renders another dimension to megasonic cleaning. Megasonic Sweeping Technology has overcomes the predominant limitation of non- uniformity of Megasonics. Instead of acoustic power being confined in PZT bonded area now the power was distributed uniformly including edges and corners of the tank.

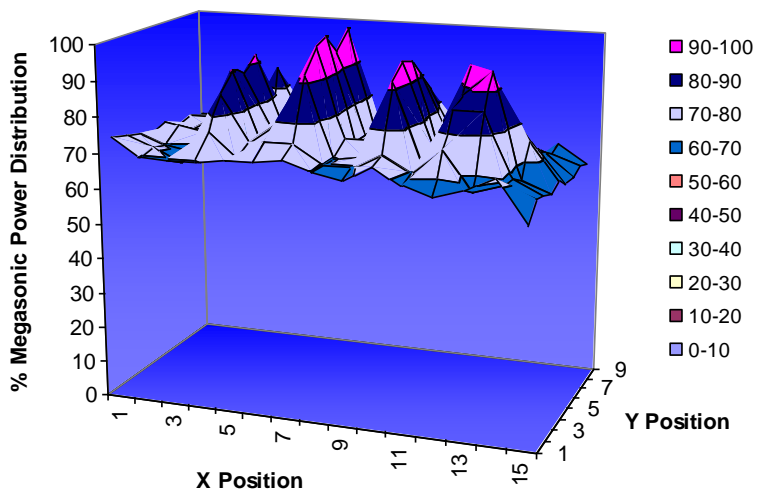

Fig. 3 Megasonic multi-frequency 360/470 kHz power distribution pattern in a cleaning vessel

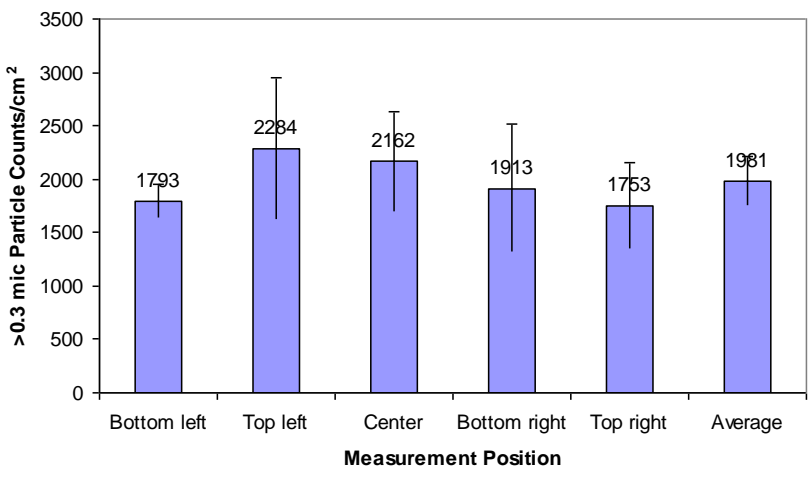

Fig. 4 Particle removal from disk drive aluminum metal component for $360 / 470 \mathrm{kHz}$

\section{B. Run to run variations of $360 \mathrm{kHz}$ Frequency Operating in Thickness Mode Transducers}

The run to run cleaning variations obtained for multifrequency operating in thickness mode transducers is shown in Figs. 5, 6. The experiments were carried out for two different types of parts such as E-coated DSP and aluminum metal spacer. The result indicates that the particle counts obtained for each run is almost same for the given experimental conditions. This is due to the fact that for each and every runs all piezoelectric transducers were operated at their optimum resonant frequency when megasonic sweeping technology is applied thus eliminates the power surges, maximize the cleaning performance and at the same time provides consistent cleaning. This is also due to the fact that in multi-frequency megasonic process the resonant frequency of the mounting plate is matched to the operating frequency of the transducers.

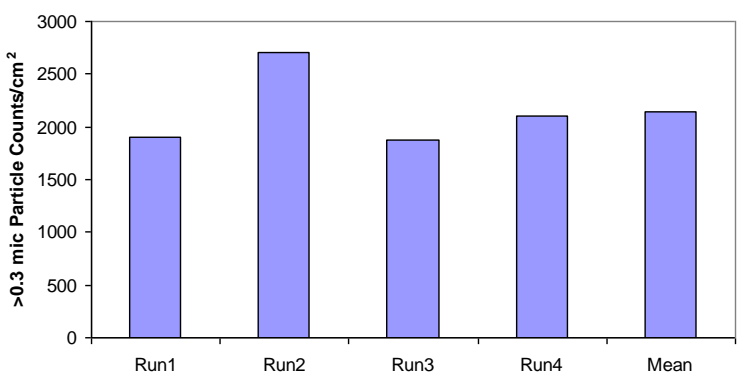

Fig. 5 Run to run cleaning variation for metal spacer 


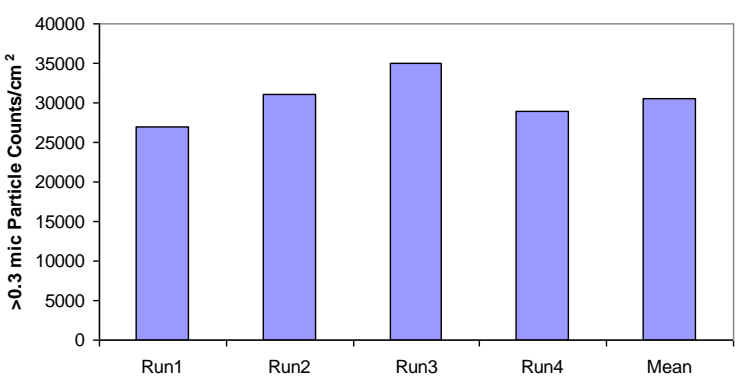

Fig. 6 Run to run cleaning variations for E-coated DSP

\section{Multiple Extraction Comparison of Various Acoustic Frequencies with Respect to Particle Removal Efficiency}

In Figs.7 -8, particle-count data are presented for an aluminum metal component, comparing the extraction efficiencies of different frequencies such as $360 \mathrm{kHz}, 470$ $\mathrm{kHz}$ and multi-frequency. The counts are shown as a function of various stages of extraction on the $\mathrm{X}$-axis. The slope of the curve indicates cleaning efficiency, and the asymptotic level indicates degree of erosion. While $360 \mathrm{kHz}$, $470 \mathrm{kHz}$ and multi-frequency exhibits the steepest initial slope, hence highest initial cleaning where as multifrequency exhibits the lowest asymptote, hence lowest erosion. From Fig. 7, it can be observed that the extracted particle counts for first stage extraction was significantly high for $360 \mathrm{kHz}$ frequency as compared to $470 \mathrm{kHz}$ and multi-frequency system. The $6^{\text {th }}$ extraction in Fig. 8 was done using $132 \mathrm{kHz}, 60 \mathrm{w} / \mathrm{gallon}$. It is to check the residual particles left on the surface based on disk drive industries component cleaning LPC procedure. From Fig. 8, it can be seen that the particle count value of $6^{\text {th }}$ extraction stage is increased for $470 \mathrm{kHz}$ whereas the particle counts value was decreased for $360 \mathrm{kHz}$ and multi-frequency. It indicates that $360 \mathrm{kHz}$ frequency and multi-frequency operating in thickness mode transducers is generating stronger acoustic cavitational force along with stronger acoustic streaming force which indeed brings down the nano-dimensional particle counts further. These combined forces in $360 \mathrm{kHz}$ frequency and multi-frequency certainly helps to enhance the removal of sub-micron and nano-dimensional particles from various components.

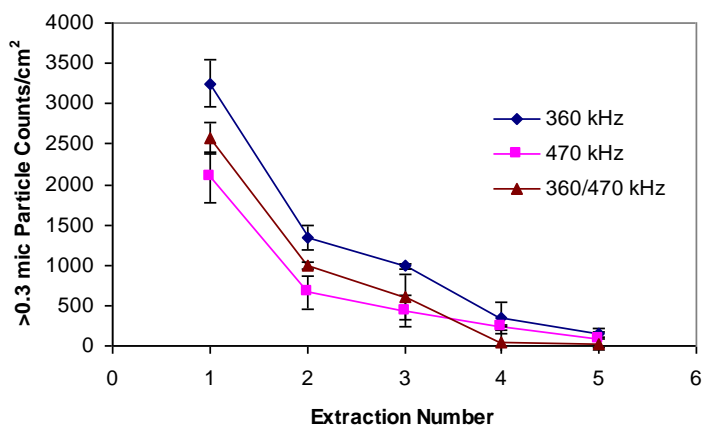

Fig. 7 Multiple-extraction comparison of various acoustic frequencies with respect to particle removal efficiency and erosion propensity for aluminum metal spacer

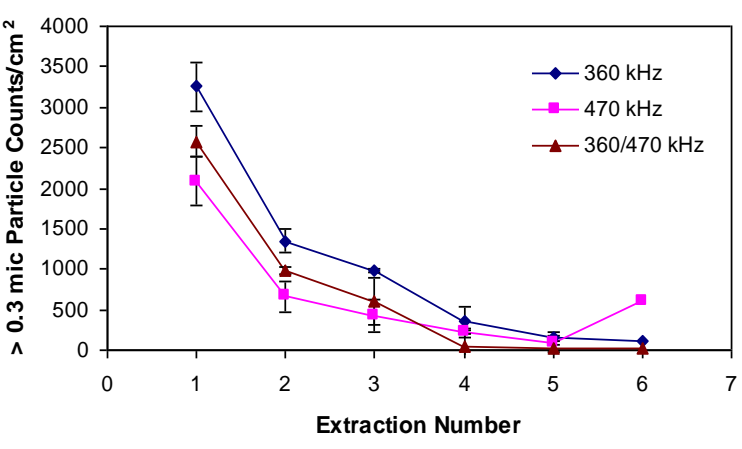

Fig. 8 Multiple-extraction comparison of various acoustic frequencies with respect to particle removal efficiency and 6th extraction was done using $132 \mathrm{kHz}, 60 \mathrm{~W} / \mathrm{gallon}$

\section{Surface cleanliness and maximum cleaning potential comparison of various acoustic frequencies}

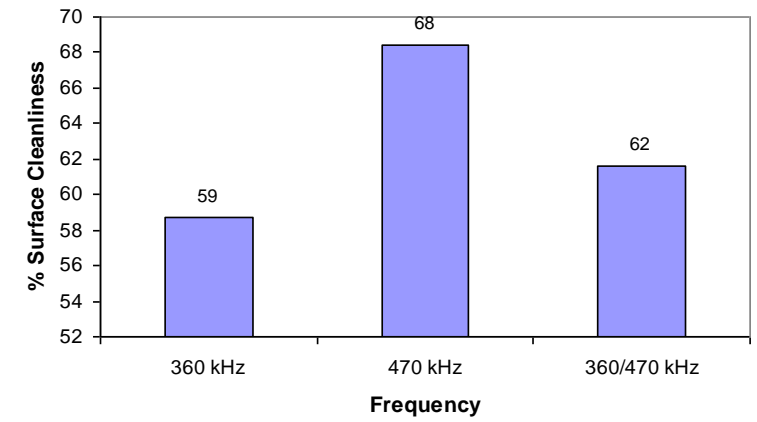

Fig. 9 Effect of various frequencies on surface cleanliness from metal parts

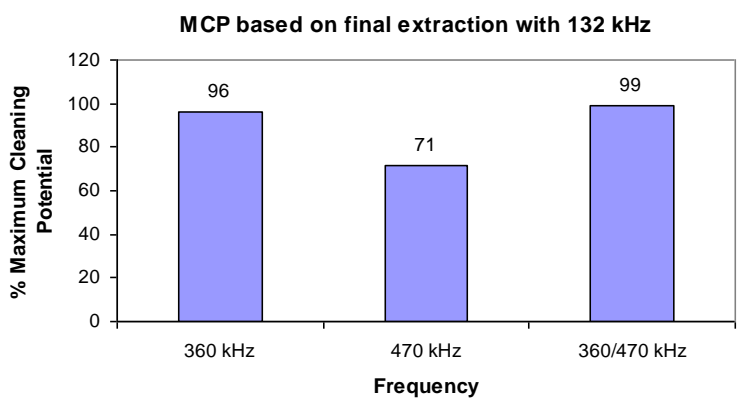

Fig. 10 Effect of various frequencies on maximum cleaning potential for metal components $\left(6^{\text {th }}\right.$ extraction with $\left.132 \mathrm{kHz}\right)$ 


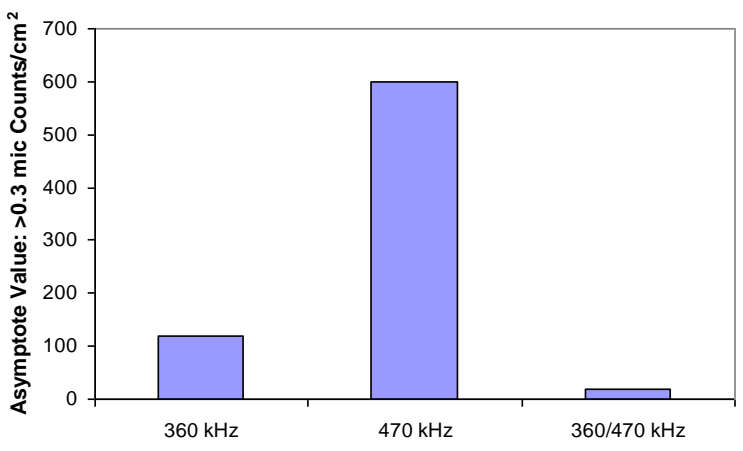

Fig. 11 Asymptote value after $6^{\text {th }}$ stage extraction with $132 \mathrm{kHz}$

The surface cleanliness, maximum cleaning potential and asymptote value obtained for various frequencies is shown in Figs. 9-11. In general the cleanability curves can be described by two different fundamental cleaning factors, the surface cleanabilty, SC and maximum cleaning potential, MCP [11]. The surface cleanabilty is a measure of the relative ease of cleaning materials during early stages of cleaning. The first stage cleaning is calculated by using this formula,

$S C=\frac{C_{0}-C_{1}}{C_{0}-C_{a}}$

Where, $\mathrm{C}_{0}$ is the initial cleanliness, $\mathrm{C}_{1}$ is the cleanliness after first extraction, and $\mathrm{C}_{\mathrm{a}}$ is the asymptotic cleanliness.

From Fig. 9, it can be observed that the surface cleaning is high for $470 \mathrm{kHz}$ and low for $360 \mathrm{kHz}$ and multifrequency.

The maximum cleaning potential (MCP) is more representative of the final cleanliness value and the MCP can be defined as follows,

Maximum Cleaning Potential $=\left[1-\frac{C_{a}}{C_{0}}\right]$

From Fig.10, it can be observed that the maximum cleaning potential is high for $360 / 470 \mathrm{kHz}$ operating with thickness mode transducers as compared to conventional single frequency $360 \mathrm{kHz}$ and $470 \mathrm{kHz}$. It indicates that the piezoelectric transducers bonded alternatively to produce multi-frequency can better match with the resonant frequency of vibrating plate and provides better cleaning results compared to conventional single frequency megasonic system.

From Fig. 11, it can be seen that the asymptote value is significantly low for multi-frequency and $360 \mathrm{kHz}$ and the asymptote value is high for $470 \mathrm{kHz}$. So, the multi- frequency megasonic system is also suitable for erosion sensitive parts.

\section{Conclusions}

The cleaning performance of multi-frequency megasonic system operating in thickness mode transducers was demonstrated successfully to remove nano-dimensional and sub-micron particles from the metal surface and the results obtained were compared with the conventional single frequency megasonic system such as $360 \mathrm{kHz}$ and $470 \mathrm{kHz}$. The maximum cleaning potential obtained for multifrequency megasonic system was almost $30 \%$ higher than $470 \mathrm{kHz}$ and almost $4 \%$ higher than $360 \mathrm{kHz}$ megasonic system. The erosion potential is also low for multifrequency megasonic system as compared to $360 \mathrm{kHz}$ and $470 \mathrm{kHz}$ system. Employing multi-frequency in the same tank can helps to remove wide ranges of particle sizes more effectively from various surfaces and also helps to improve overall yield. Future study involves collecting more data's using multi-frequency megasonic system and finds the application for various fields.

\section{Acknowledgment}

We wish to thank Tjung (Managing Director), Beng Hooi (Manager Sales Dept), and Research and Development team (ACT, Penang) for their kind support for this project.

\section{References}

[1] Vetrimurugan et al., "Experimental Investigation of Ultrasonic and Megasonic Frequency on Cleaning of Various Disk Drive Components", International Journal of Chemical Engineering and Applications", Vol.4, No.4, pp. 174-177, 2013.

[2] Goodson, J.M., and Nagarajan, R., "Megasonic Sweeping and Silicon Wafer Cleaning", Solid State Phenomena, Vol. 145-156, pp. 27-30, 2009.

[3] McQueen, D.H., "Frequency dependence of ultrasonic cleaning", Ultrasonics, Vol. 24, pp. 273-280, 1986

[4] Nagarajan et al., "Megasonic cleaning to remove nano-dimensional contaminants from wafer surfaces: An analytical study", Solid State Phenomena, Vol.195, pp.209-212, 2013

[5] R. Nagarajan, R. W. Welker, and R. L. Weaver, Evaluation of aqueous cleaning techniques for disk drive parts, Microcontamination Conference Proceedings, San Jose, CA, Oct. 1991; 16-18: 312-326.

[6] H. Kim, Y.Lee and E.Lim, "A multi-frequency megasonic system for nano-particle removal", 24 $4^{\text {th }}$ Electochemical Society Meeting, 2013.

[7] Vetrimurugan, R., and Beng Hooi, N.G., "Study of ultrasonic parameters on removal of contamination from slider surface by using various cleaning chemistry", International Journal of Chemical and Environmental Engineering, Vol. 3, pp. 392-396, 2012.

[8] Roger, W.W., Nagarajan, R., and Newberg, C.E., "Contamination and ESD control in High-Technology Manufacturing", IEEE press, John Wiley and Sons, New Jersey, ch. 5, pp. 195-230, 2006.

[9] Goodson, J.M, Megasonic multi-frequency apparatus with matched transducers and mounting plate, patent no: US9108232 B2, 2015.

[10] Goodson, J. M., and Skillman, "Megasonic Processing Apparatus With Frequency Sweeping of Thickness Mode Transducers", US Patent, Patent No. US 8,310,131 B2, pp. 1-13, 2012.

[11] Nagarajan, R, and Welker, R.W, Size distributions of particles extracted from disk drive parts: comparison with the MIL-STD-1246 distribution, Journal of the Institute of Environmental Science and Technology, Vol. 43, 4, pp. 25-31, 2000. 
Proc. of The Fourth Intl. Conf. On Advances in Applied Science and Environmental Technology - ASET 2016

Copyright ( $)$ Institute of Research Engineers and Doctors, USA .All rights reserved.

ISBN: 978-1-63248-097-2 doi: 10.15224/ 978-1-63248-097-2-44

\section{Biography}

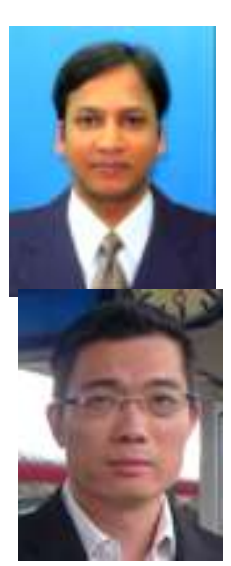

Name: Vetrimurugan, Crest Ultrasonics.

Name: Lim Teong Kheng (Terry), Crest Ultrasonics.

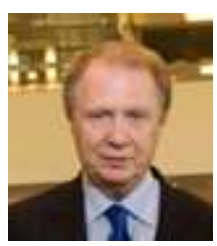

Name: J. Michael Goodson, Crest Ultrasonics.

Name: Sebastian Thottathil, Crest Ultrasonics 